\title{
Hémodissection Pariétale Oblitérante des Vaisseaux Intracrâniens, une Forme Particulière d'Anévrysme Disséquant
}

\author{
F. GAGNE, M. LEMAY et S. VERRET
}

RÉSUMÉ: Une fillette de six ans décède rapidement des suites d'une obstruction artérielle sylvienne droite survenue inopinément. L'autopsie révèle un clivage de la paroi sylvienne droite avec introduction de sang entre la limitante élastique interne et la média, et thrombose secondaire.

SUMMARY: $A$ six year old girl complained of sudden severe headache, became hemiplegic and unconscious. A right carotid arteriogram revealed an obstruction of the right anterior cerebral artery and many sylvian branches. Death occurred four days later. At autopsy, a recent softening of nearly all the right middle cerebral arterial territory was found. Thrombus filled the sylvian artery and its main branches. Histologic examination of the vessel walls showed a dissecting infiltration of blood between
Cette forme particulière d'anévrysme disséquent constitue une entité nosologique bien caractéristique qu'on a appelée "Hémodissection pariétale oblitérante des vaisseaux intracraniens." Elle atteint des sujets jeunes et sa cause précise demeure inconnue.

the internal elastic lamina and the media. This particular form of dissecting aneurysm, occurring in young subjects, in the absence of atherosclerosis, high blood pressure and idiopathic medial necrosis, represents a distinct nosologic entity that has been called "Obstructive parietal hemodissection of intracranial vessels." The pathogenesis of the disease is unknown: trauma has been mentioned, also congenital defects in the elastic lamina or other morphologic abnormalities of that lamina.
Des départements de Pathologie et de Neurologie de l'Hôpital de l'Enfant-Jésus, Québec, Québec, Canada.

Les demandes de tirés-à-part devront être adressées au Dr. F. Gagné, Département de Pathologie, Hôpital de l'Enfant-Jésus, 1401, 18e Rue, Québec, Québec G1J 1Z4 Canada.

From the Departments of Pathology and Neurology of the Hospital Infant Jesus, Quebec, Quebec, Canada.

Reprint requests to Dr. F. Gagne, Department of Pathology, Hospital Infant Jesus, 1401, 18th St., Quebec, Quebec G1J 1Z4, Canada.
Ce terme a été récemment proposé par Perier et al., (1966) pour caractériser une forme particulière d'anévrysme disséquant intracrânien survenant sans cause connue chez des sujets jeunes. Le clivage de la paroi vasculaire s'effectue entre la lame élastique interne, et la média et non en pleine couche musculaire comme dans les anévrysmes disséquants habituellement décrits. L'observation qui suit constitue une illustration typique de la maladie: elle s'accompagne d'une brève revue de la littérature et de quelques hypothèses concernant la pathogénie de la lésion.

\section{OBSERVATION CLINIQUE}

L. Q. (H.E.J.: A 174893), fillette de six ans, droitière sans antécédent pathologique, revenant du terrain de jeu le 29 juillet 1974 , se plaint brusquement d'une violente céphalée. Elle tombe sans pouvoir se relever, demeure consciente mais parle avec difficulté. Pendant qu'on la transporte à l'hôpital, elle vomit à une reprise. Au questionnaire, les parents rapportent une toux légère depuis une couple de jour. A la salle d'urgence, l'enfant est somnolente mais bien orientée; la nuque est souple et les pupilles égales, moyennes, réagissant à la lumière. Le fond d'oeil est normal; on constate une hémianopsie homonyme gauche, une paralysie faciale gauche de type central, une déviation du voile du palais, une dysarthrie modérée, une hémiparésie gauche proportionnelle et une hémianesthésie gauche. Le réflexe cutané plantaire est en extension à gauche. Les deux carotides sont pulsatiles, sans souffle. La température rectale est normale à 
$99.3^{\circ} \mathrm{F}$., la tension artérielle à $100 / 60$ et le rythme cardiaque régulier à 70/minute. Le reste de l'examen physique est sans particularité.

A la ponction lombaire, la pression initiale du liquide céphalorachidien est de $22 \mathrm{~cm} \mathrm{H} \mathrm{H}^{2} \mathrm{O}$ et finale de $18 \mathrm{~cm} \mathrm{H}^{2} \mathrm{O}$; la cytologie et l'analyse biochimique du liquide sont normales. Des radiographies du crâne, de la colonne cervicale et des poumons sont également normales. L'échoencéphalogramme ne démontre pas de déviation des structures de la ligne médiane.

Une artériographie carotidienne droite pratiquée par cathétérisation fémorale donne le résultat suivant: la carotide interne droite de même que le siphon carotidien sont libres mais il y a obstruction complète de la cérébrale antérieure, de la partie moyenne de la sylvienne, d'une branche temporale et d'une branche frontale de cette même sylvienne. Les vaisseaux thalamostriés demeurent perméables.

Les examens suivants faits en cours d'hospitalisation sont normaux: électrocardiogramme, formule sanguine, glycémie, bilan électrolytique, examen d'urine, sédimentation globulaire, azotémie, C.P.K., S.G.O.T., S.G.P.T., temps de Quick, temps de céphaline et décompte des plaquettes. La re- cherche des antistreptolysines est négative de même que la culture des sécrétions pharyngées et l'hémoculture.

Le 31 juillet à 19.00 heures, l'enfant est stuporeuse; la pupille droite est plus grande que la gauche. La nuque est toujours souple. Le réflexe cutané-plantaire est en extension des deux côtés. On administre par voie intraveineuse 10 $\mathrm{mg}$ de dexaméthasone et $200 \mathrm{cc}$ de mannitol à 20\%. A 22.30 heures, l'enfant est semi-comateuse; la pupille droite est en mydriase fixe. L.e premier août à 02.30 heures, il se produit un arrêt cardio-respiratoire; l'enfant est réanimée, intubée et ventilée artificiellement. Malgré le dexaméthasone intraveineux et le glycérol $75 \%$ donné par sonde nasogastrique, les signes d'hypertension intra-crânienne persistent. Par la suite il s'installe un coma profond et la tension artérielle chute malgré les vasopresseurs. Le 2 août 1974 après deux électroencéphalogrammes ne révélant qu'une ligne isoélectrique, les manoeuvres de soutien sont cessées et le décès est constaté à 14.00 heures.

A l'examen post-mortem, les viscères du tronc ne présentent qu'un petit infarctus rénal récent mal explicable ainsi que des lésions pul- monaires récentes de pneumonite chimique par aspiration du contenu gastrique. Les organes intracrâniens pésent $1,590 \mathrm{~g}$; les hémisphères cérébraux sont aplatis et le tissu est en général plutôt mou. L'hémisphère droit est plus gros que le gauche. On remarque aussi un peu de congestion veineuse méningée. La sylvienne droite est de calibre moyen et sa lumière contient du sang coagulé. A la coupe, la ligne médiane est déviée vers la gauche et les ventricules sont passablement collabés. A l'hémisphère droit la substance blanche est abondante et le tissu est mollasse dans la presque totalité du territoire sylvien. Dans cette même région, il y a une zone de $2.5 \mathrm{~cm}$ environ où le cortex est de coloration rougeâtre.

A l'examen histologique la large lésion cérébrale sylvienne décrite montre des images dégénératives cellulaires et de l'oedème réalisant l'aspect caractéristique d'un infarctus très récent. La glande hypophysaire est aussi intéressée par un processus nécrosant diffus. L'examen de l'artère sylvienne droite et de ses principales branches montre un clivage net et très étendu de l'intima' et de la limitante élastique interne, avec infiltration de sang entre ces structures et la média, entraînant la thrombose (Fig. 1).

\section{2.}

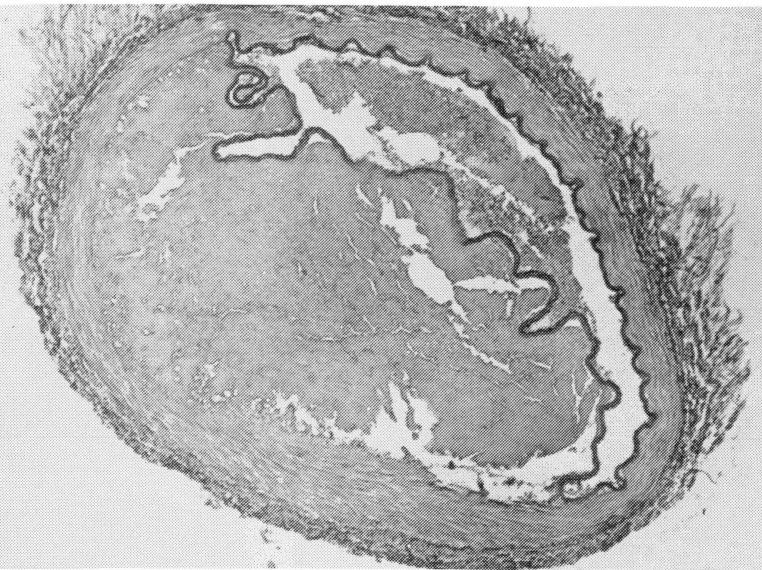

Figure 1-Coupe transversale de l'artère sylvienne droite montrant la limitante élastique interne réfléchie sur elle-même et très largement clivée par rapport à la média artérielle. La lumière pré-existante est réduite à une fente latérale par l'accumulation de sang fraîchement coagulé dans l'espace produit par le clivage. Col. de Weigert, pour les fibres elastiques (X 150).

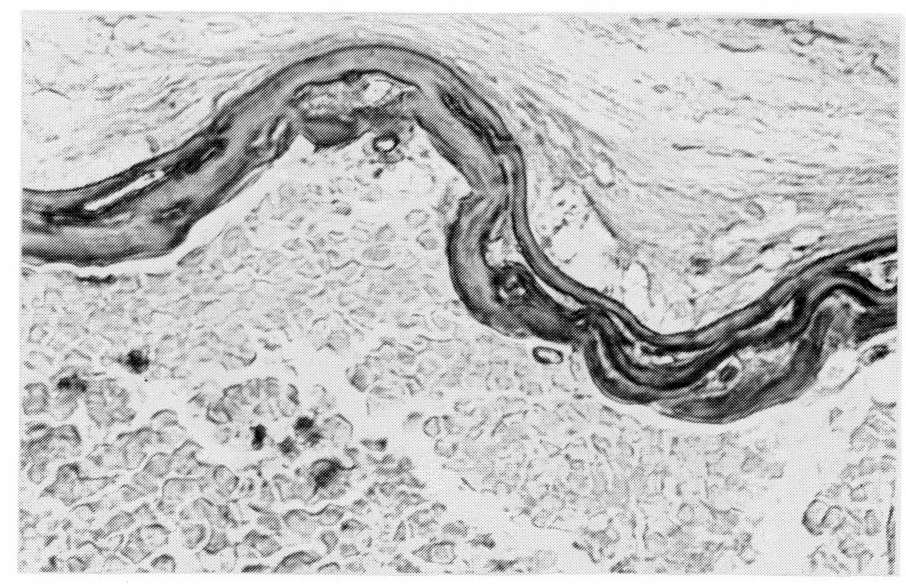

Figure 2-Fort grossissement de la membrane limitante élastique interne à la toute proximité de la zone de clivage pariétal, montrant l'épaisseur exagérée de cette membrane avec son caractère irrégulièrement nodulaire et feuilleté. Ebauche de clivage sous la membrane. Col. de Weigert (X 1500). 


\section{DISCUSSION}

Il s'agit donc d'une oblitération vasculaire sylvienne due à un anévrysme disséquant particulier par la localisation du clivage entre la limitante élastique et la média, et non au voisinage du tiers interne de la média comme dans les anévrysmes disséquants habituels reliés à l'athéromatose, à l'hypertension artérielle et à la médianécrose idiopathique.

Parmi ces étiologies, seule la média-nécrose pourrait s'envisager chez notre enfant, en relation avec le syndrome de Marfan. Or, dans ce cas comme dans tous ceux colligés par Dourov et al., (1975) on ne retrouve pas le phénotype du syndrome de Marfan (stature longiligne, arachnodactylie), ni la luxation du cristallin, ni une malformation cardiaque. Il n'existe par ailleurs pas aux parois vasculaires de lésions histologiques de média-nécrose.

Cette maladie n'est pas fréquente: elle frappe de façon subite et imprévisible des sujets jeunes et en bonne santé. A la suite de Perier et al., (1966); Dourov et al., (1975) font un revue complète de la question et identifient ce syndrome comme une entité nosologique nouvelle sous le titre d'hémodissection pariétale intracrânienne oblitérante. Ces derniers auteurs retrouvent dans la littérature 26 observations bien documentées auxquelles ils ajoutent un cas personnel. A ces 27 cas il faut ajouter ceux d'Adelman et al., (1974); de Chang et al., (1975); de Hochberg et al., (1975) et le nôtre pour un total de 31 observations.

La pathogénie de la maladie est inconnue. On a souvent tenté d'in- criminer un traumatisme (Dourov et al., 1975; Stehbens, 1972) mais les accidents reliés à la maladie ont toujours été très bénins et insuffisants pour expliquer la lésion. Le plus souvent il n'y a d'ailleurs aucun traumatisme récent. Plusieurs auteurs (Wolman, 1959; Adelman et al., 1974; Escourolle et al., 1972; et Dourov et Flament-Durand, 1975) rapportent des absences localisées vraisemblablement congénitales de la limitante élastique interne; ces points faibles pariétaux pourraient se prêter à l'infiltration hémorragique. Dans notre cas, nous n'avons pas retrouvé de telles lésions pariétales. Comme Escourolle et al., (1972) et Chang et al., (1975), cependant, nous avons remarqué dans nos coupes prevenant de l'artère malade des zones importantes où la limitante élastique nous paraît épassie, un peu nodulaire, percée de fissures longitudinales par endroits même multilamellaire (Fig. 2). Comme ces auteurs nous avons retrouvé dans des vaisseaux témoins provenant du même enfant et d'autres enfants de même age, des altérations du même ordre mais indiscutablement beaucoup moins accusées. Ces altérations ne sont pas présentes partout dans les zones lésionnelles. Les lames élastiques anormales, colorées par le trichrome de Masson apparaissent sous forme de membranes hyalines colorées en rouge d'une façon un peu nodulaire avec, surtout en surface, des zones prenant le bleu d'aniline. Parfois, sur une lame élastique à peu près normale on remarque un mince dépôt collagénique.
Ces modifications de la lame élastique sont-elles à l-origine de la dissection ou en sont-elles la conséquence? Quelle est leur nature exacte? Il ne nous paraît pas possible de le définir actuellement. Peutil enfin exister une relation entre ce type de lésion et l'anévrysme disséquant conventionnel? Seul le cas d'Escourolle et al., (1972) survenu chez un sujet de 32 ans associait la dissection intra-médiale au clivage sous intimal artériel.

\section{BIBLIOGRAPHIE}

ADELMAN, L. S., DOE, F. D. and SARNAT, H. B. (1974). Bilateral Dissecting Aneurysm of the Internal Carotid Arteries. Acta Neuropath. (Berlin), 29: 93-97

CHANG, V., REWCASTLE, N B., HARWOOD-NASH, D. C. F. and NORMAN, M. G. (1975). Bilateral Dissecting Aneurysm of the Intracranial Carotid Arteries in a 8-year-old boy. Neurology, 25: 573-579.

DOUROV, N et FLAMENT-DURAND, J (1975). L'hémodissection pariétale oblitérante des vaisseaux intra-crâniens chez le sujet jeune. Annales d'Anatomie Pathologique (Paris), 20: 7-22.

ESCOUROLLE, R., GAUTHIER, J.-C., ROSA, A., DER AGOPIAN, P. et LHERMITTE, F (1972). Anévrysme disséquant vertébro-basilaire. Rev Neurol. 128: 95-104.

HOCHBERG, F H., BEAN, C., FISHER, C. M. and ROBERSON, G. H. (1975). Stroke in a 15-year-old girl secondary to terminal Carotid Dissection. Neurology 25: 725-729.

PERIER, O., BRIHAYE, J. et DHAENE, R. (1966). Hémodissection pariétale oblitérante ("anévrysme disséquant") de l'artère basilaire. Acta Neurol. belg. 66: 123-141.

STEHBENS, W. E. (1972). Pathology of the Cerebral Blood Vessels. The C. V. Mosby Company (St-Louis), 440-445.

WOLMAN, L. (1959). Cerebral Dissecting Aneurysm. Brain, 82: 276-291. 